\title{
EXTENSIONS OF THE RESULTS ON POWERS OF p-HYPONORMAL AND log-HYPONORMAL OPERATORS
}

\author{
CHANGSEN YANG AND JIANGTAO YUAN
}

Received 22 November 2004; Revised 27 April 2005; Accepted 10 May 2005

Firstly, we will show the following extension of the results on powers of $p$-hyponormal and log-hyponormal operators: let $n$ and $m$ be positive integers, if $T$ is $p$-hyponormal for $p \in(0,2]$, then: (i) in case $m \geq p,\left(T^{n+m^{*}} T^{n+m}\right)^{(n+p) /(n+m)} \geq\left(T^{n^{*}} T^{n}\right)^{(n+p) / n}$ and $\left(T^{n} T^{n^{*}}\right)^{(n+p) / n} \geq\left(T^{n+m} T^{n+m^{*}}\right)^{(n+p) /(n+m)}$ hold, (ii) in case $m<p, \quad T^{n+m^{*}} T^{n+m} \geq$ $\left(T^{n^{*}} T^{n}\right)^{(n+m) / n}$ and $\left(T^{n} T^{n^{*}}\right)^{(n+m) / n} \geq T^{n+m} T^{n+m^{*}}$ hold. Secondly, we will show an estimation on powers of $p$-hyponormal operators for $p>0$ which implies the best possibility of our results. Lastly, we will show a parallel estimation on powers of log-hyponormal operators as follows: let $\alpha>1$, then the following hold for each positive integer $n$ and $m$ : (i) there exists a log-hyponormal operator $T$ such that $\left(T^{n+m^{*}} T^{n+m}\right)^{n \alpha /(n+m)} \nsucceq\left(T^{n^{*}} T^{n}\right)^{\alpha}$,

(ii) there exists a log-hyponormal operator $T$ such that $\left(T^{n} T^{n^{*}}\right)^{\alpha} \nsupseteq\left(T^{n+m} T^{n+m^{*}}\right)^{n \alpha /(n+m)}$.

Copyright (c) 2006 C. Yang and J. Yuan. This is an open access article distributed under the Creative Commons Attribution License, which permits unrestricted use, distribution, and reproduction in any medium, provided the original work is properly cited.

\section{Introduction}

In this paper, let $H$ be a complex Hilbert space and $B(H)$ be the algebra of all bounded linear operators in $H$, and a capital letter mean an element of $B(H)$. An operator $T$ is said to be positive (in symbol: $T \geq 0$ ) if $(T x, x) \geq 0$ for any $x \in H$, and an operator $T$ is said to be strictly positive (in symbol: $T>0$ ) if $T$ is positive and invertible.

An operator $T$ is said to be $p$-hyponormal for $p>0$ if $\left(T^{*} T\right)^{p} \geq\left(T T^{*}\right)^{p}$, where $T^{*}$ is the adjoint operator of $T$. An invertible operator $T$ is said to be log-hyponormal if $\log \left(T^{*} T\right) \geq \log \left(T T^{*}\right)$. If $p=1, T$ is called hyponormal and if $p=1 / 2, T$ is called semihyponormal. It is clear that every $p$-hyponormal operator is $q$-hyponormal for $0<q \leq p$ by the celebrated Löwner-Heinz theorem and every invertible $p$-hyponormal operator for $p>0$ is log-hyponormal since $\log t$ is an operator monotone function. log-hyponormality is sometimes regarded as 0 -hyponormal since $\left(X^{p}-1\right) / p \rightarrow \log X$ as $p \rightarrow 0$ for $X>0$.

Recently, Furuta-Yanagida [6] showed the following results on powers of $p$-hyponormal operators with $p \in(0,1]$ which is a generalization of Aluthge-Wang [1]. 
2 Powers of $p$ - and log-hyponormal operators

Theorem 1.1 [6]. Let $T$ be a p-hyponormal operator for $p \in(0,1]$. Then

$$
\begin{aligned}
& \left(T^{n^{*}} T^{n}\right)^{(p+1) / n} \geq \cdots \geq\left(T^{2^{*}} T^{2}\right)^{(p+1) / 2} \geq\left(T^{*} T\right)^{p+1}, \\
& \left(T T^{*}\right)^{p+1} \geq\left(T^{2} T^{2^{*}}\right)^{(p+1) / 2} \geq \cdots \geq\left(T^{n} T^{n^{*}}\right)^{(p+1) / n}
\end{aligned}
$$

hold for all positive integer $n$.

Very recently, Ito [8] showed that Theorem 1.1 holds for $p>0$.

Theorem 1.2 [8]. If $T$ is $p$-hyponormal for $p \in(k-1, k]$ where $k$ is a positive integer, then

$$
\left(T^{1+m^{*}} T^{1+m}\right)^{(1+p) /(1+m)} \geq\left(T^{*} T\right)^{1+p}, \quad\left(T T^{*}\right)^{1+p} \geq\left(T^{1+m} T^{1+m^{*}}\right)^{(1+p) /(1+m)}
$$

hold for all positive integer $m$ such that $m \geq p$,

$$
T^{1+m^{*}} T^{1+m} \geq\left(T^{*} T\right)^{1+m}, \quad\left(T T^{*}\right)^{1+m} \geq T^{1+m} T^{1+m^{*}}
$$

hold for all positive integer $m$ such that $m<p$.

Yamazaki [13] also showed the following Theorem 1.3 which is a parallel result to Theorems 1.1 and 1.2.

Theorem 1.3 [13]. If $T$ is log-hyponormal, then

$$
\left(T^{n+1^{*}} T^{n+1}\right)^{n /(n+1)} \geq T^{n^{*}} T^{n}, \quad T^{n} T^{n^{*}} \geq\left(T^{n+1} T^{n+1^{*}}\right)^{n /(n+1)}
$$

hold for all positive integer $n$.

We can rewrite Theorem 1.3 into the following easily.

Theorem 1.4. If $T$ is log-hyponormal, then

$$
\left(T^{n+m^{*}} T^{n+m}\right)^{n /(n+m)} \geq T^{n^{*}} T^{n}, \quad T^{n} T^{n^{*}} \geq\left(T^{n+m} T^{n+m^{*}}\right)^{n /(n+m)}
$$

hold for all positive integer $n$ and $m$.

In fact, if $m>1$, then by Theorem 1.3 we have

$$
\begin{gathered}
\left|T^{n+m}\right|^{2(n+m-1) /(n+m)} \geq\left|T^{n+m-1}\right|^{2}, \ldots,\left|T^{n+2}\right|^{2(n+1) /(n+2)} \geq\left|T^{n+1}\right|^{2}, \\
\left|T^{n+1}\right|^{2 n /(n+1)} \geq\left|T^{n}\right|^{2}, \\
\left|T^{n^{*}}\right|^{2} \geq\left|T^{n+1^{*}}\right|^{2 n /(n+1)}, \\
\left|T^{n+1^{*}}\right|^{2} \geq\left|T^{n+2^{*}}\right|^{2(n+1) /(n+2)}, \ldots,\left|T^{n+m-1^{*}}\right|^{2} \geq\left|T^{n+m^{*}}\right|^{2(n+m-1) /(n+m)},
\end{gathered}
$$


so that

$$
\begin{aligned}
& \left(T^{n+m^{*}} T^{n+m}\right)^{n /(n+m)} \geq\left(T^{n+m-1^{*}} T^{n+m-1}\right)^{n /(n+m-1)} \geq \cdots \geq\left(T^{n+1^{*}} T^{n+1}\right)^{n /(n+1)} \geq T^{n^{*}} T^{n}, \\
& T^{n} T^{n^{*}} \geq\left(T^{n+1} T^{n+1^{*}}\right)^{n /(n+1)} \geq \cdots \geq\left(T^{n+m-1} T^{n+m-1^{*}}\right)^{n /(n+m-1)} \geq\left(T^{n+m} T^{n+m^{*}}\right)^{n /(n+m)}
\end{aligned}
$$

hold by Löwner-Heinz inequality.

In this paper, we will show Theorem 2.1, the parallel result to Theorem 1.4, stated below which is an extension of Theorems 1.1 and 1.2. We will also show an estimation on powers of $p$-hyponormal operators for $p>0$ which implies the best possibility of Theorem 2.1 and discuss the best possibility of Theorem 1.4.

\section{An extension of Theorems 1.1 and 1.2}

Theorem 2.1. If $T$ is $p$-hyponormal for $p \in(k-1, k]$ where $k$ is a positive integer, then

(i) in case $k=1,2$,

$$
\begin{aligned}
& \left(T^{n+m^{*}} T^{n+m}\right)^{(n+p) /(n+m)} \geq\left(T^{n^{*}} T^{n}\right)^{(n+p) / n}, \\
& \left(T^{n} T^{n^{*}}\right)^{(n+p) / n} \geq\left(T^{n+m} T^{n+m^{*}}\right)^{(n+p) /(n+m)}
\end{aligned}
$$

hold for all positive integer $n$ and $m$ such that $m \geq p$;

(ii) in case $k=2,3$,

$$
\begin{aligned}
& T^{n+m^{*}} T^{n+m} \geq\left(T^{n^{*}} T^{n}\right)^{(n+m) / n}, \\
& \left(T^{n} T^{n^{*}}\right)^{(n+m) / n} \geq T^{n+m} T^{n+m^{*}}
\end{aligned}
$$

hold for all positive integer $n$ and $m$ such that $m<p$.

Corollary 2.2. Let $n$ and $m$ be positive integers, if $T$ is $p$-hyponormal for $p \in(k-1, k]$, then

(i) in case $k=1,2$ and $m \geq p$,

$$
\begin{aligned}
& \left(T^{n+m^{*}} T^{n+m}\right)^{(n+p) /(n+m)} \geq \cdots \geq\left(T^{n+k^{*}} T^{n+k}\right)^{(n+p) /(n+k)} \geq\left(T^{n^{*}} T^{n}\right)^{(n+p) / n}, \\
& \left(T^{n} T^{n^{*}}\right)^{(n+p) / n} \geq\left(T^{n+k^{*}} T^{n+k}\right)^{(n+p) /(n+k)} \geq \cdots \geq\left(T^{n+m} T^{n+m^{*}}\right)^{(n+p) /(n+m)} ;
\end{aligned}
$$

(ii) in case $k=3$,

$$
\begin{aligned}
& \left(T^{n+2^{*}} T^{n+2}\right)^{(n+1) /(n+2)} \geq T^{n+1^{*}} T^{n+1} \geq\left(T^{n^{*}} T^{n}\right)^{(n+1) / n}, \\
& \left(T^{n} T^{n^{*}}\right)^{(n+1) / n} \geq T^{n+1} T^{n+1^{*}} \geq\left(T^{n+2} T^{n+2^{*}}\right)^{(n+1) /(n+2)} .
\end{aligned}
$$

Remark 2.3. In case $p \in(0,1]$, Theorem 1.1 follows from Corollary 2.2(i) by taking $k=1$ and $n=1$.

In case $k=1,2,3$ and $p \in(k-1, k]$, Theorem 1.2 follows from Theorem 2.1 by taking $n=1$.

We need the following results to show Theorem 2.1. 
4 Powers of $p$ - and log-hyponormal operators

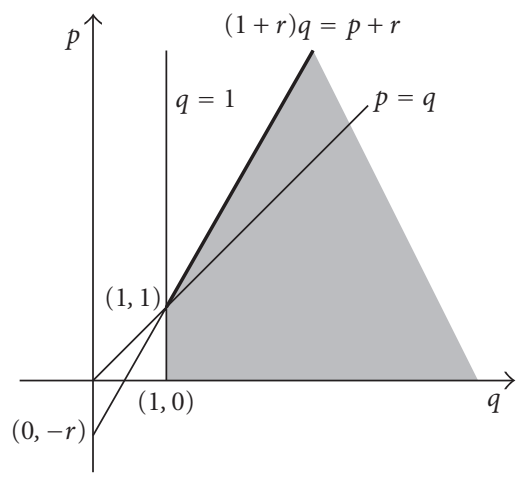

Figure 2.1 The best possible domain for Furuta inequality.

Theorem 2.4 (Furuta inequality [3] call it FI simply). If $A \geq B \geq 0$, then for each $r \geq 0$,

(i)

$$
\left(B^{r / 2} A^{p} B^{r / 2}\right)^{1 / q} \geq\left(B^{r / 2} B^{p} B^{r / 2}\right)^{1 / q},
$$

(ii)

$$
\left(A^{r / 2} A^{p} A^{r / 2}\right)^{1 / q} \geq\left(A^{r / 2} B^{p} A^{r / 2}\right)^{1 / q}
$$

hold for $p \geq 0$ and $q \geq 1$ with $(1+r) q \geq p+r$.

FI yields the following famous Löwner-Heinz inequality by putting $r=0$ in (i) or (ii) of FI. It was shown by Tanahashi [11] that the domain drawn for $p, q$ and $r$ in Figure 2.1 is the best possible for FI.

Theorem 2.5 Löwner-Heinz (Löner-Heinz inequality $[7,10]$ call it L-H simply). Let $1 \geq$ $\alpha \geq 0$. Then $A \geq B \geq 0 \Rightarrow A^{\alpha} \geq B^{\alpha}$.

Lemma 2.6 [5]. Let $\alpha \in R$ and $X$ be invertible. Then $\left(X^{*} X\right)^{\alpha}=X^{*}\left(X X^{*}\right)^{\alpha-1} X$ holds, especially in case $\alpha \geq 1$ Lemma 2.6 holds without invertibility of $X$.

Theorem $2.7[2,4,5,9]$. Let $A, B \geq 0$ such that $A^{\alpha} \geq B^{\alpha}$ for $\alpha>0$. Then for each $q \geq 0$ and $t \geq 0$ the following hold.

(1) $f_{t, q}(s)=\left(A^{t / 2} B^{s} A^{t / 2}\right)^{(q+t) /(s+t)}$ is decreasing for $s \geq q \geq 0$.

(2) $g_{t, q}(s)=\left(B^{t / 2} A^{s} B^{t / 2}\right)^{(q+t) /(s+t)}$ is increasing for $s \geq q \geq 0$.

Theorem 2.8 [13]. Let $T$ be a p-hyponormal operator for $p \in(0,1]$. Then

$$
\begin{aligned}
& \left(T^{n^{*}} T^{n}\right)^{1 / n} \geq \cdots \geq\left(T^{2^{*}} T^{2}\right)^{1 / 2} \geq T^{*} T, \\
& T T^{*} \geq\left(T^{2} T^{2^{*}}\right)^{1 / 2} \geq \cdots \geq\left(T^{n} T^{n^{*}}\right)^{1 / n}
\end{aligned}
$$

hold for all positive integer $n$. 
Lemma 2.9. If $T$ is $p$-hyponormal for $p \in(k-1, k]$ where $k$ is a positive integer, then

$$
\begin{array}{r}
\left(\left|T^{*}\right|\left|T^{n+m}\right|^{2}\left|T^{*}\right|\right)^{(n+1+p) /(n+1+m)} \geq\left|T^{*}\right|\left|T^{n+m}\right|^{2(n+p) /(n+m)}\left|T^{*}\right| \\
\left(|T|\left|T^{n+m^{*}}\right|^{2}|T|\right)^{(n+1+p) /(n+1+m)} \leq|T|\left|T^{n+m^{*}}\right|^{2(n+p) /(n+m)}|T|
\end{array}
$$

hold for all positive integer $n$ and $m$ such that $m \geq k$. If $k=1,2$ in addition, then

$$
\begin{gathered}
\left|T^{*}\right|\left|T^{n}\right|^{2(n+p) / n}\left|T^{*}\right| \geq\left(\left|T^{*}\right|\left|T^{n}\right|^{2}\left|T^{*}\right|\right)^{(n+1+p) /(n+1)}, \\
|T|\left|T^{n^{*}}\right|^{2(n+p) / n}|T| \leq\left(|T|\left|T^{n^{*}}\right|^{2}|T|\right)^{(n+1+p) /(n+1)}
\end{gathered}
$$

hold for all positive integer $n$.

Proof of Lemma 2.9. Put $\gamma=\min \{1, p\}$, then

$$
\left(T^{n^{*}} T^{n}\right)^{\gamma / n} \geq \cdots \geq\left(T^{2^{*}} T^{2}\right)^{\gamma / 2} \geq\left(T^{*} T\right)^{\gamma} \geq\left(T T^{*}\right)^{\gamma} \geq\left(T^{2} T^{2^{*}}\right)^{\gamma / 2} \geq \cdots \geq\left(T^{n} T^{n^{*}}\right)^{\gamma / n}
$$

holds by Theorem 2.8, p-hyponormality of T and L-H.

Proof of (2.12). Since $\left(\left|T^{n+m}\right|^{2 /(n+m)}\right)^{\gamma} \geq\left(\left|T^{*}\right|^{2}\right)^{\gamma}$ by (2.16), then for each $t \geq 0$ and $q \geq 0$, $g_{t, q}(s)=\left(\left|T^{*}\right|^{t}\left|T^{n+m}\right|^{2 s /(n+m)}\left|T^{*}\right|^{t}\right)^{(q+t) /(s+t)}$ is increasing for $s \geq q \geq 0$ by Theorem $2.7(2)$. Then by taking $\alpha=\gamma, t=1, q=n+p$ and $s=n+m$ we have

$$
\begin{aligned}
\left(\left|T^{*}\right|\left|T^{n+m}\right|^{2}\left|T^{*}\right|\right)^{(n+1+p) /(n+1+m)} \\
=\left(\left|T^{*}\right|\left|T^{n+m}\right|^{2(n+m) /(n+m)}\left|T^{*}\right|\right)^{(n+1+p) /(n+1+m)} \\
=g_{1, n+p}(n+m) \\
\quad \geq g_{1, n+p}(n+p) \\
=\left(\left|T^{*}\right|\left|T^{n+m}\right|^{2(n+p) /(n+m)}\left|T^{*}\right|\right)^{(n+p+1) /(n+p+1)} \\
=\left|T^{*}\right|\left|T^{n+m}\right|^{2(n+p) /(n+m)}\left|T^{*}\right| .
\end{aligned}
$$

Proof of (2.13). Since $\left(|T|^{2}\right)^{\gamma} \geq\left(\left|T^{n+m^{*}}\right|^{2 /(n+m)}\right)^{\gamma}$ by (2.16), similar to the proof of (2.12), (2.13) holds by taking $\alpha=\gamma, t=1, q=n+p$ and $s=n+m$ in Theorem 2.7(1).

Proof of (2.14). If $k=1,2$, then $p \in(0,1]$ or $p \in(1,2]$, thus $\gamma+n \geq \gamma+1 \geq p$. So that $(1+n / \gamma)((1+n) / p) \geq 1 / \gamma+n / \gamma$ holds.

On the other hand, by applying (ii) of Theorem 2.4 to $\left|T^{n}\right|^{2 \gamma / n}$ and $\left|T^{*}\right|^{2 \gamma}$ for $(1+$ $n / \gamma)((1+n) / p) \geq 1 / \gamma+n / \gamma$, we have

$$
\begin{aligned}
\left|T^{n}\right|^{2 p / n} & =\left|T^{n}\right|^{(2 \gamma / n)(p / \gamma)} \\
& \geq\left(\left|T^{n}\right|^{(2 \gamma / n)(n / 2 \gamma)}\left|T^{*}\right|^{2 \gamma(1 / \gamma)}\left|T^{n}\right|^{(2 \gamma / n)(n / 2 \gamma)}\right)^{p /(n+1)} \\
& =\left(\left|T^{n}\right|\left|T^{*}\right|^{2}\left|T^{n}\right|\right)^{p /(n+1)},
\end{aligned}
$$


6 Powers of $p$ - and log-hyponormal operators

so that

$$
\begin{aligned}
\left|T^{*}\right|\left|T^{n}\right|^{2(n+p) / n}\left|T^{*}\right| & =\left|T^{*}\right|\left|T^{n}\right|\left|T^{n}\right|^{2 p / n}\left|T^{n}\right|\left|T^{*}\right| \\
& \geq\left|T^{*}\right|\left|T^{n}\right|\left(\left|T^{n}\right|\left|T^{*}\right|^{2}\left|T^{n}\right|\right)^{p /(n+1)}\left|T^{n}\right|\left|T^{*}\right| \\
& =\left(\left|T^{*}\right|\left|T^{n}\right|^{2}\left|T^{*}\right|\right)^{(n+1+p) /(n+1)} .
\end{aligned}
$$

Proof of (2.15). Since $|T|^{2 \gamma} \geq\left|T^{n^{*}}\right|^{2 \gamma / n}$ by (2.16), similar to the proof of (2.14), (2.15) holds by Lemma 2.6 and taking $p_{1}=1 / \gamma, r_{1}=n / \gamma$ and $q_{1}=(1+n) / p$ in Theorem 2.4(i).

Proof of Theorem 2.1. Let $T=U|T|$ be the polar decomposition of $T$. Then it is well known that the polar decomposition of $T^{*}$ is $T^{*}=U^{*}\left|T^{*}\right|$.

Proof of (2.1). In case $k=1,2$.

(i) We will prove that the following (2.20) holds for all positive integer $n$ by induction:

$$
\left(T^{n+k^{*}} T^{n+k}\right)^{(n+p) /(n+k)} \geq\left(T^{n^{*}} T^{n}\right)^{(n+p) / n}
$$

Firstly, we prove that (2.20) holds for $n=1$, that is, in case $k=1, p \in(0,1]$, then

$$
\left(T^{2^{*}} T^{2}\right)^{(1+p) / 2} \geq\left(T^{*} T\right)^{p+1}
$$

and in case $k=2, p \in(1,2]$, then

$$
\left(T^{3^{*}} T^{3}\right)^{(1+p) / 3} \geq\left(T^{*} T\right)^{p+1}
$$

In case $k=1, p \in(0,1]$, since $\left(T^{*} T\right)^{p} \geq\left(T T^{*}\right)^{p}$, by applying (i) of Theorem 2.4 to $\left(T^{*} T\right)^{p}$ and $\left(T T^{*}\right)^{p}$ for $(1+(1 / p))((1+1) /(1+p)) \geq 1 / p+1 / p$, we have

$$
\begin{aligned}
\left(T^{1+1^{*}} T^{1+1}\right)^{(1+p) /(1+1)} & =\left(U^{*}\left|T^{*}\right| T^{*} T\left|T^{*}\right| U\right)^{(1+p) /(1+1)} \\
& =U^{*}\left(\left(\left|T^{*}\right|^{2 p}\right)^{1 / 2 p}\left(|T|^{2 p}\right)^{1 / p}\left(\left|T^{*}\right|^{2 p}\right)^{1 / 2 p}\right)^{(1+p) /(1+1)} U \\
& \geq U^{*}\left(\left|T^{*}\right|^{2 p}\right)^{(1+p) / p} U \\
& =U^{*}\left|T^{*}\right|^{2(1+p)} U \\
& =|T|^{2(1+p)} \\
& =\left(T^{*} T\right)^{1+p}
\end{aligned}
$$

so that $(2.21)$ is proved.

In case $k=2$, if $T$ is $p$-hyponormal for $p \in(1,2]$, then $T$ is hyponormal (i.e., 1-hyponormal) by L-H; thus $T^{2^{*}} T^{2} \geq\left(T^{*} T\right)^{2}$ by $(2.21)$, so $\left(T^{2^{*}} T^{2}\right)^{p / 2} \geq\left(T^{*} T\right)^{p} \geq\left(T T^{*}\right)^{p}$ by $p$-hyponomality of $T$ and $\mathrm{L}-\mathrm{H}$ for $p / 2 \in(0,1]$, we have the following by applying (i) 
of Theorem 2.4 to $\left(T^{2^{*}} T^{2}\right)^{p / 2}$ and $\left(T T^{*}\right)^{p}$ for $(1+1 / p)((1+2) /(1+p)) \geq 2 / p+1 / p$ :

$$
\begin{aligned}
\left(T^{1+2^{*}} T^{1+2}\right)^{(1+p) /(1+2)} & =\left(U^{*}\left|T^{*}\right| T^{2^{*}} T^{2}\left|T^{*}\right| U\right)^{(1+p) /(1+2)} \\
& =U^{*}\left(\left(\left|T^{*}\right|^{2 p}\right)^{1 / 2 p}\left(\left|T^{2}\right|^{p}\right)^{2 / p}\left(\left|T^{*}\right|^{2 p}\right)^{1 / 2 p}\right)^{(1+p) /(1+2)} U \\
& \geq U^{*}\left(\left|T^{*}\right|^{2 p}\right)^{(1+p) / p} U \\
& =U^{*}\left|T^{*}\right|^{2(1+p)} U \\
& =|T|^{2(1+p)} \\
& =\left(T^{*} T\right)^{1+p},
\end{aligned}
$$

so that $(2.22)$ is proved.

Secondly, assumed that $(2.20)$ holds for $1, \ldots, n(\geq 1)$. We will prove that $(2.20)$ holds for $n+1$.

In fact, we have

$$
\begin{aligned}
\left(T^{n+1+k^{*}} T^{n+1+k}\right)^{(n+1+p) /(n+1+k)} & =\left(T^{*}\left|T^{n+k}\right|^{2} T\right)^{(n+1+p) /(n+1+k)} \\
& =\left(U^{*}\left|T^{*}\right|\left|T^{n+k}\right|^{2}\left|T^{*}\right| U\right)^{(n+1+p) /(n+1+k)} \\
& =U^{*}\left(\left|T^{*}\right|\left|T^{n+k}\right|^{2}\left|T^{*}\right|\right)^{(n+1+p) /(n+1+k)} U \\
& \geq U^{*}\left|T^{*}\right|\left|T^{n+k}\right|^{2(n+p) /(n+k)}\left|T^{*}\right| U \quad \text { by }(2.12) \\
& \geq U^{*}\left|T^{*}\right|\left|T^{n}\right|^{2(n+p) /(n)}\left|T^{*}\right| U \text { by induction } \\
& \geq U^{*}\left(\left|T^{*}\right|\left|T^{n}\right|^{2}\left|T^{*}\right|\right)^{(n+1+p) /(n+1)} U \text { by }(2.14) \\
& =\left(T^{*}\left|T^{n}\right|^{2} T\right)^{(n+1+p) /(n+1)} \\
& =\left(T^{n+1^{*}} T^{n+1}\right)^{(n+1+p) /(n+1)}
\end{aligned}
$$

so that it is proved that (2.20) (i.e., case $m=k$ of (2.1)) holds for $n+1$.

(ii) We will prove that $(2.1)$ holds for $m>k$.

If $k=1, p \in(0,1]$, then $m>1$ and by (2.20) we have

$$
\begin{aligned}
\left(T^{n+m^{*}} T^{n+m}\right)^{(n+m-1+p)(n+m)} & \geq\left(T^{n+m-1^{*}} T^{n+m-1}\right)^{(n+m-1+p) /(n+m-1)}, \ldots, \\
\left(T^{n+2^{*}} T^{n+2}\right)^{(n+1+p) /(n+2)} & \geq\left(T^{n+1^{*}} T^{n+1}\right)^{(n+1+p) /(n+1)}, \\
\left(T^{n+1^{*}} T^{n+1}\right)^{(n+p) /(n+1)} & \geq\left(T^{n^{*}} T^{n}\right)^{(n+p) / n},
\end{aligned}
$$


8 Powers of $p$ - and log-hyponormal operators

thus by L-H we have

$$
\begin{aligned}
\left(T^{n+m^{*}} T^{n+m}\right)^{(n+p) /(n+m)} & \geq\left(T^{n+m-1^{*}} T^{n+m-1}\right)^{(n+p) /(n+m-1)} \geq \cdots \\
& \geq\left(T^{n+1^{*}} T^{n+1}\right)^{(n+p) /(n+1)} \geq\left(T^{n^{*}} T^{n}\right)^{(n+p) / n} .
\end{aligned}
$$

If $k=2, T$ is $p$-hyponormal for $p \in(1,2]$, then $T$ is hyponormal (i.e., 1-hyponormal) by L-H; thus

$$
T^{n+1^{*}} T^{n+1} \geq\left(T^{n^{*}} T^{n}\right)^{(n+1) / n}
$$

holds by case $k=1$ and $p=1$ of (2.20), so we have

$$
\begin{aligned}
T^{n+m^{*}} T^{n+m} & \geq\left(T^{n+m-1^{*}} T^{n+m-1}\right)^{(n+m) /(n+m-1)}, \ldots, \\
T^{n+3^{*}} T^{n+3} & \geq\left(T^{n+2^{*}} T^{n+2}\right)^{(n+3) /(n+2)}, \\
\left(T^{n+2^{*}} T^{n+2}\right)^{(n+p) /(n+2)} & \geq\left(T^{n^{*}} T^{n}\right)^{(n+p) / n}
\end{aligned}
$$

by (2.28) and (2.20) (the last inequality holds by (2.20)), so that the following holds by L-H:

$$
\begin{aligned}
\left(T^{n+m^{*}} T^{n+m}\right)^{(n+p) /(n+m)} & \geq\left(T^{n+m-1^{*}} T^{n+m-1}\right)^{(n+p) /(n+m-1)} \\
& \geq \cdots \geq\left(T^{n+2^{*}} T^{n+2}\right)^{(n+p) /(n+2)} \geq\left(T^{n^{*}} T^{n}\right)^{(n+p) / n} .
\end{aligned}
$$

Consequently, the proof of (2.1) is complete by combining (i) and (ii).

Proof of (2.2). The proof is similar to the proof of (2.1), so we omit it here.

Proof of (2.3). If $k=2$, we only need to show $T^{n+1^{*}} T^{n+1} \geq\left(T^{n^{*}} T^{n}\right)^{(n+1) / n}$, this is just (2.28), so that (2.3) holds for $k=2$.

If $k=3$, we need to show $T^{n+1^{*}} T^{n+1} \geq\left(T^{n^{*}} T^{n}\right)^{(n+1) / n}$ and $T^{n+2^{*}} T^{n+2} \geq\left(T^{n^{*}} T^{n}\right)^{(n+2) / n}$. In fact $T$ is $p$-hyponormal for $p \in(2,3]$, then $T$ is 1-hyponormal by $L$ - $H$; thus $T^{n+1^{*}} T^{n+1} \geq\left(T^{n^{*}} T^{n}\right)^{(n+1) / n}$ holds by case $k=1$ and $p=1$ of $(2.1)$; similarly, $T$ is $p$ hyponormal for $p \in(2,3]$, then $T$ is 2-hyponormal by $\mathrm{L}-\mathrm{H}$; thus $T^{n+2^{*}} T^{n+2} \geq$ $\left(T^{n^{*}} T^{n}\right)^{(n+2) / n}$ holds by case $k=2, p=2$ and $m=2$ of (2.1), so that (2.3) holds.

Proof of (2.4). The proof is similar to that of (2.3), so we omit it here.

Proof of Corollary 2.2. We have (i) by the process of the proof of (2.1) and (2.2). (ii) is obvious by case $k=3$ of (2.3), (2.4) and L-H. 


\section{Estimation on powers of $p$-hyponormal and log-hyponormal operators}

The following Theorem 3.1 which is an estimation on powers of $p$-hyponormal operators for $p>0$ implies the best possibility of Theorem 2.1.

Theorem 3.1. Let $k$, $n$ and $m$ be positive integers, $p \in(k-1, k]$ and $\alpha>1$.

(1) In case $m \geq p$ the following hold.

(i) There exists a p-hyponormal operator $T$ such that

$$
\left(T^{n+m^{*}} T^{n+m}\right)^{(n+p) \alpha /(n+m)} \nsucceq\left(T^{n^{*}} T^{n}\right)^{(n+p) \alpha / n} .
$$

(ii) There exists a p-hyponormal operator T such that

$$
\left(T^{n} T^{n^{*}}\right)^{(n+p) \alpha / n} \nsupseteq\left(T^{n+m} T^{n+m^{*}}\right)^{(n+p) \alpha / n+m} .
$$

(2) In case $m<p$ the following hold.

(i) There exists a p-hyponormal operator $T$ such that

$$
\left(T^{n+m^{*}} T^{n+m}\right)^{\alpha} \nsucceq\left(T^{n^{*}} T^{n}\right)^{(n+m) \alpha / n} .
$$

(ii) There exists a p-hyponormal operator $T$ such that

$$
\left(T^{n} T^{n^{*}}\right)^{(n+m) \alpha / n} \nsucceq\left(T^{n+m} T^{n+m^{*}}\right)^{\alpha} .
$$

The following Theorem 3.2 which is a parallel result to Theorem 3.1 implies the best possibility of Theorem 1.4.

TheOREM 3.2. Let $\alpha>1$. Then the following hold for each positive integer $n$ and $m$.

(i) There exists a log-hyponormal operator $T$ such that $\left(T^{n+m^{*}} T^{n+m}\right)^{n \alpha /(n+m)} \nsupseteq\left(T^{n^{*}} T^{n}\right)^{\alpha}$.

(ii) There exists a log-hyponormal operator $T$ such that $\left(T^{n} T^{n^{*}}\right)^{\alpha} \nsupseteq\left(T^{n+m} T^{n+m^{*}}\right)^{n \alpha /(n+m)}$.

We need the following results to show Theorems 3.1 and 3.2.

Theorem $3.3[12,14]$. Let $\delta>0, p>0, r>0$, and $q>0$. If $0<q<1$ or $(\delta+r) q<p+r$, then the following assertions hold.

(i) There exist positive invertible operators $A$ and $B$ on $R^{2}$ such that

$$
A^{\delta} \geq B^{\delta}, \quad\left(B^{r / 2} A^{p} B^{r / 2}\right)^{1 / q} \nsucceq B^{(p+r) / q} .
$$

(ii) There exist positive invertible operators $A$ and $B$ on $R^{2}$ such that

$$
A^{\delta} \geq B^{\delta}, \quad A^{(p+r) / q} \nsupseteq\left(A^{r / 2} B^{p} A^{r / 2}\right)^{1 / q} .
$$


Powers of $p$ - and log-hyponormal operators

Theorem 3.4 [14]. Let $p>0, r>0$, and $q>0$. If $r q<p+r$, then the following assertions hold.

(i) There exist positive invertible operators $A$ and $B$ on $R^{2}$ such that

$$
\log A \geq \log B, \quad\left(B^{r / 2} A^{p} B^{r / 2}\right)^{1 / q} \nsucceq B^{(p+r) / q} .
$$

(ii) There exist positive invertible operators $A$ and $B$ on $R^{2}$ such that

$$
\log A \geq \log B, \quad A^{(p+r) / q} \nsupseteq\left(A^{r / 2} B^{p} A^{r / 2}\right)^{1 / q} .
$$

Lemma 3.5. For positive operators $A$ and $B$ on $H$, define an operator $T$ on $\bigoplus_{k=-\infty}^{\infty} H_{k}$ where $H_{k} \cong H$ as follows:

$$
\left(\begin{array}{ccccccc}
\ddots & & & & & & \\
\ddots & 0 & & & & & \\
& B^{1 / 2} & 0 & & & & \\
& & B^{1 / 2} & (0) & & & \\
& & & A^{1 / 2} & 0 & & \\
& & & & A^{1 / 2} & 0 & \\
& & & & & \ddots & \ddots
\end{array}\right) \text {, }
$$

where $(\cdot)$ shows the place of the $(0,0)$ matrix element. Then the following assertions hold.

(i) $T$ is $p$-hyponormal for $p>0$ if and only if $A^{p} \geq B^{p}$.

(ii) $T$ is $\log$-hyponormal if and only if $A$ and $B$ are invertible and $\log A \geq \log B$.

Furthermore, the following assertions hold for $\beta>0$ and any positive integer $n$ and $m$ :

(iii) $\left(T^{n+m^{*}} T^{n+m}\right)^{\beta /(n+m)} \geq\left(T^{n^{*}} T^{n}\right)^{\beta / n}$ if and only if

$$
\begin{aligned}
& \left(B^{l / 2} A^{n+m-l} B^{l / 2}\right)^{\beta /(n+m)} \geq\left(B^{l / 2} A^{n-l} B^{l / 2}\right)^{\beta / n} \quad \text { holds for } l=1,2, \ldots, n-1 . \\
& \left(B^{l / 2} A^{n+m-l} B^{l / 2}\right)^{\beta /(n+m)} \geq B^{\beta} \quad \text { holds for } l=n, n+1, \ldots, n+m-1 .
\end{aligned}
$$

(iv) $\left(T^{n} T^{n^{*}}\right)^{\beta / n} \geq\left(T^{n+m} T^{n+m^{*}}\right)^{\beta /(n+m)}$ if and only if

$$
\begin{gathered}
\left(A^{j / 2} B^{n-j} A^{j / 2}\right)^{\beta / n} \geq\left(A^{j / 2} B^{n+m-j} A^{j / 2}\right)^{\beta /(n+m)} \quad \text { holds for } j=1,2, \ldots, n-1 . \\
A^{\beta} \geq\left(A^{j / 2} B^{n+m-j} A^{j / 2}\right)^{\beta /(n+m)} \quad \text { holds for } j=n, n+1, \ldots, n+m-1 .
\end{gathered}
$$


C. Yang and J. Yuan 11

Proof. By easy calculation, we have

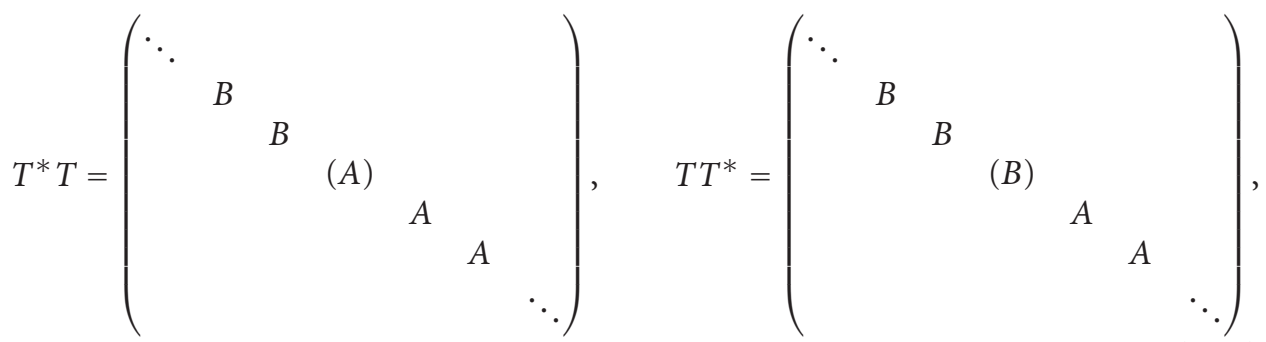

so that (i) is obvious by comparing the two $(0,0)$ elements of $\left(T^{*} T\right)^{p}$ and $\left(T T^{*}\right)^{p}$, similarly, (ii) is obvious by comparing the two $(0,0)$ elements of $\log T^{*} T$ and $\log T T^{*}$. Furthermore, the following hold for $n \geq 2$ :

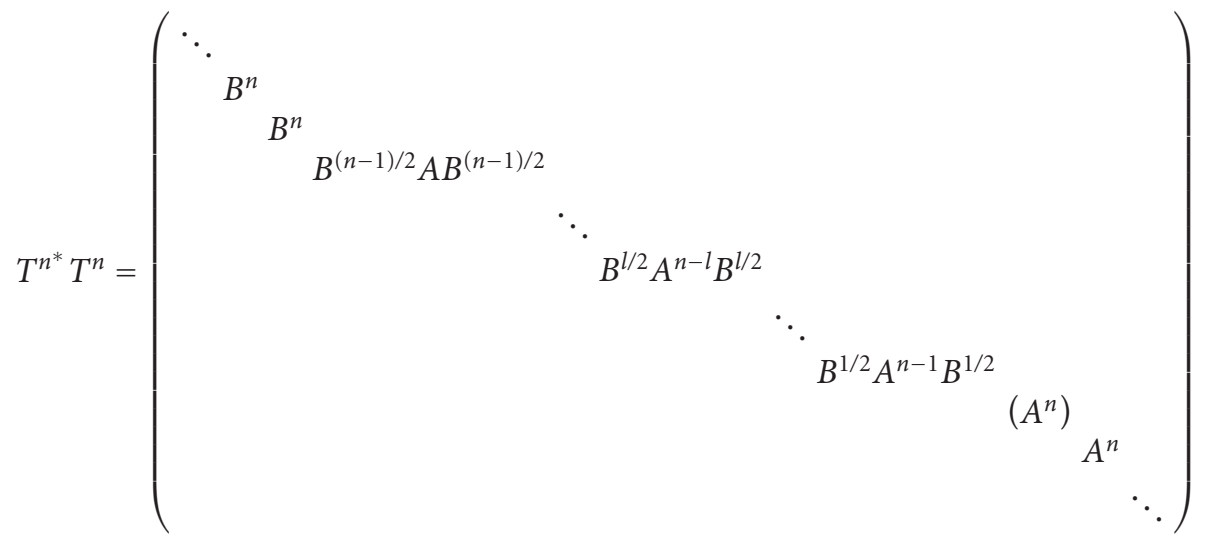

so that we have (iii) by comparing the corresponding elements of $\left(T^{n+m^{*}} T^{n+m}\right)^{\beta /(n+m)}$ and $\left(T^{n^{*}} T^{n}\right)^{\beta / n}$,

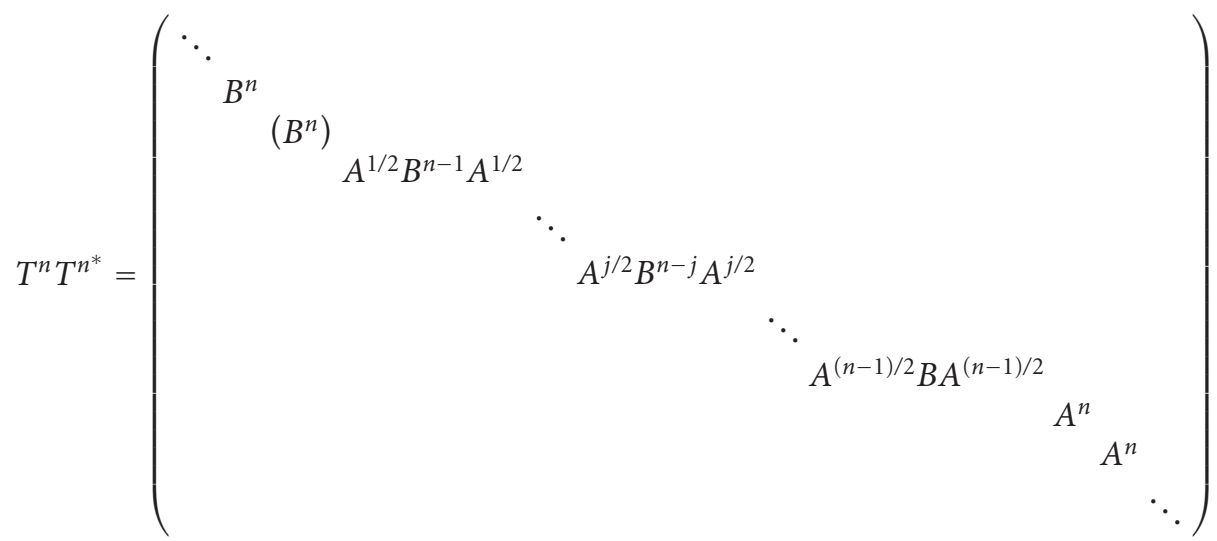


so that we have (iv) by comparing the corresponding elements of $\left(T^{n} T^{n^{*}}\right)^{\beta / n}$ and $\left(T^{n+m} T^{n+m^{*}}\right)^{\beta /(n+m)}$.

Proof of Theorem 3.1. Put $p_{1}=m>0, r_{1}=n>0, q_{1}=(n+m) /\left(n+p_{0}\right) \alpha$ where $p_{0}=$ $\min \{p, m\}$, and $\delta=p>0$, then we have $q_{1}<1$ when $m<p ;\left(\delta+r_{1}\right) q_{1}=(n+p)((n+$ $m) /(n+p) \alpha)<n+m=p_{1}+r_{1}$ when $m \geq p$.

By (i) of Theorem 3.3, there exist positive invertible operators $A$ and $B$ on $R^{2}$ such that $A^{\delta} \geq B^{\delta}$ and $\left(B^{r_{1} / 2} A^{p_{1}} B^{r_{1} / 2}\right)^{1 / q_{1}} \nsucceq B^{\left(p_{1}+r_{1}\right) / q_{1}}$, that is,

$$
\begin{gathered}
A^{p} \geq B^{p}, \\
\left(B^{n / 2} A^{m} B^{n / 2}\right)^{\left(n+p_{0}\right) \alpha /(n+m)} ¥ B^{\left(n+p_{0}\right) \alpha} .
\end{gathered}
$$

Define an operator $T$ on $\bigoplus_{k=-\infty}^{\infty} H_{k}$ where $H_{k} \cong R^{2}$ as (3.9). Then $T$ is $p$-hyponormal by (3.15) and (i) of Lemma 3.5, and $\left(T^{n+m^{*}} T^{n+m}\right)^{\left(n+p_{0}\right) \alpha /(n+m)} ¥\left(T^{n^{*}} T^{n}\right)^{\left(n+p_{0}\right) \alpha / n}$ by (iii) of Lemma 3.5 since the case $l=n$ of (3.10) does not hold for $\beta=\left(n+p_{0}\right) \alpha$ by (3.16), so that Theorems 3.1(1)(i) and 3.1(2)(i) hold.

By (ii) of Theorem 3.3, there exist positive invertible operators $A$ and $B$ on $R^{2}$ such that $A^{\delta} \geq B^{\delta}$ and $A^{\left(p_{1}+r_{1}\right) / q_{1}} \nsucceq\left(A^{r_{1} / 2} B^{p_{1}} A^{r_{1} / 2}\right)^{1 / q_{1}}$, that is,

$$
\begin{gathered}
A^{p} \geq B^{p}, \\
A^{\left(n+p_{0}\right) \alpha} \nsupseteq\left(A^{n / 2} B^{m} A^{n / 2}\right)^{\left(n+p_{0}\right) \alpha /(n+m) .}
\end{gathered}
$$

Define an operator $T$ on $\bigoplus_{k=-\infty}^{\infty} H_{k}$ where $H_{k} \cong R^{2}$ as (3.9). Then $T$ is $p$-hyponormal by (3.17) and (i) of Lemma 3.5, and $\left(T^{n} T^{n^{*}}\right)^{\left(n+p_{0}\right) \alpha / n} ¥\left(T^{n+m} T^{n+m^{*}}\right)^{\left(n+p_{0}\right) \alpha /(n+m)}$ by (iv) of Lemma 3.5 since the case $j=n$ of (3.11) does not hold for $\beta=\left(n+p_{0}\right) \alpha$ by (3.18), so that Theorems 3.1(1)(ii) and 3.1(2)(ii) hold.

Proof of Theorem 3.2. Put $p_{1}=m>0, r_{1}=n>0, q_{1}=(n+m) / n \alpha$, then we have $r_{1} q_{1}=$ $(n+m) / \alpha<n+m=p_{1}+r_{1}$.

By (i) of Theorem 3.4, there exist positive invertible operators $A$ and $B$ on $R^{2}$ such that

$$
\log A \geq \log B
$$

and $\left(B^{r_{1} 2} A^{p_{1}} B^{r_{1} / 2}\right)^{1 / q_{1}} \nsucceq B^{\left(p_{1}+r_{1}\right) / q_{1}}$, that is,

$$
\left(B^{n / 2} A^{m} B^{n / 2}\right)^{n \alpha /(n+m)} \nsucceq B^{n \alpha}
$$

Define an operator $T$ on $\bigoplus_{k=-\infty}^{\infty} H_{k}$ where $H_{k} \cong R^{2}$ as (3.9). Then $T$ is log-hyponormal by (3.19) and (ii) of Lemma 3.5, and $\left(T^{n+m^{*}} T^{n+m}\right)^{n \alpha /(n+m)} \nsucceq\left(T^{n^{*}} T^{n}\right)^{n \alpha / n}$ by (iii) of Lemma 3.5 since the case $l=n$ of (3.10) does not hold for $\beta=n \alpha$ by (3.20), so that Theorem 3.2(i) holds. 
By (ii) of Theorem 3.4, there exist positive invertible operators $A$ and $B$ on $R^{2}$ such that

$$
\log A \geq \log B
$$

and $A^{\left(p_{1}+r_{1}\right) / q_{1}} \nsupseteq\left(A^{r_{1} / 2} B^{p_{1}} A^{r_{1} / 2}\right)^{1 / q_{1}}$, that is,

$$
A^{n \alpha} \nsucceq\left(A^{n / 2} B^{m} A^{n / 2}\right)^{n \alpha /(n+m)} .
$$

Define an operator $T$ on $\bigoplus_{k=-\infty}^{\infty} H_{k}$ where $H_{k} \cong R^{2}$ as (3.9). Then $T$ is log-hyponormal by (3.21) and (ii) of Lemma 3.5, and $\left(T^{n} T^{n^{*}}\right)^{n \alpha / n} \nsucceq\left(T^{n+m} T^{n+m^{*}}\right)^{n \alpha /(n+m)}$ by (iv) of Lemma 3.5 since the case $j=n$ of (3.11) does not hold for $\beta=n \alpha$ by (3.22), so that Theorem 3.2 (ii) and holds.

\section{Acknowledgments}

We would like to express our cordial gratitude to the referee for his valuable advice and suggestions. This work was supported in part by Education Foundation of Henan Province (2003110006).

\section{References}

[1] A. Aluthge and D. Wang, Powers of p-hyponormal operators, Journal of Inequalities and Applications 3 (1999), no. 3, 279-284.

[2] M. Fujii, T. Furuta, and E. Kamei, Furuta's inequality and its application to Ando's theorem, Linear Algebra and its Applications 179 (1993), 161-169.

[3] T. Furuta, $A \geqslant B \geqslant 0$ assures $\left(B^{r} A^{p} B^{r}\right)^{1 / q} \geqslant B^{\frac{p+2 r}{q}}$ for $r \geqslant 0, p \geqslant 0, q \geqslant 1$ with $(1+2 r) q \geqslant p+2 r$, Proceedings of the American Mathematical Society 101 (1987), no. 1, 85-88.

[4] _ Applications of order preserving operator inequalities, Operator Theory and Complex Analysis (Sapporo, 1991), Oper. Theory Adv. Appl., vol. 59, Birkhäuser, Basel, 1992, pp. 180190.

[5] Extension of the Furuta inequality and Ando-Hiai log-majorization, Linear Algebra and its Applications 219 (1995), 139-155.

[6] T. Furuta and M. Yanagida, On powers of p-hyponormal and log-hyponormal operators, Journal of Inequalities and Applications 5 (2000), no. 4, 367-380.

[7] E. Heinz, Beiträge zur Störungstheorie der Spektralzerlegung, Mathematische Annalen 123 (1951), 415-438 (German).

[8] M. Ito, Generalizations of the results on powers of p-hyponormal operators, Journal of Inequalities and Applications 6 (2001), no. 1, 1-15.

[9] E. Kamei, A satellite to Furuta's inequality, Mathematica Japonica 33 (1988), no. 6, 883-886.

[10] K. Löwner, Über monotone Matrixfunktionen, Mathematische Zeitschrift 38 (1934), no. 1, 177216 (German).

[11] K. Tanahashi, Best possibility of the Furuta inequality, Proceedings of the American Mathematical Society 124 (1996), no. 1, 141-146.

[12] The best possibility of the grand Furuta inequality, Proceedings of the American Mathematical Society 128 (2000), no. 2, 511-519.

[13] T. Yamazaki, Extensions of the results on p-hyponormal and log-hyponormal operators by Aluthge and Wang, SUT Journal of Mathematics 35 (1999), no. 1, 139-148. 
14 Powers of $p$ - and log-hyponormal operators

[14] M. Yanagida, Some applications of Tanahashi's result on the best possibility of Furuta inequality, Mathematical Inequalities \& Applications 2 (1999), no. 2, 297-305.

Changsen Yang: College of Mathematics and Information Science, Henan Normal University, Xinxiang 453007, China

E-mail address: yangchangsen0991@sina.com

Jiangtao Yuan: College of Mathematics and Information Science, Henan Normal University, Xinxiang 453007, China

E-mail address: yuanjiangtao02@yahoo.com.cn 